\title{
TP53 mutation and MET amplification in circulating tumor DNA analysis predict disease progression in patients with advanced gastric cancer
}

\author{
Jia Li ${ }^{\text {Equal first author, } 1,2}$, Zhaoyan $\mathbf{L i}^{\text {Equal first author, } 3}{ }^{3}$, Yajie Ding ${ }^{1}$, Yan Xu ${ }^{1}$, Xiaohong Zhu ${ }^{1}$, Nida Cao ${ }^{1}$, Chen Huang ${ }^{4}$, \\ Mengmeng Qin ${ }^{1}$, Feng Liu ${ }^{1}$, Aiguang Zhao ${ }^{\text {Corresp. } 1}$ \\ ${ }^{1}$ Department of Oncology, Longhua Hospital, Shanghai University of Traditional Chinese Medicine (TCM), Shanghai, China \\ 2 Department of Integrated Chinese and Western Medicine, Affiliated Cancer Hospital of Zhengzhou University and Henan Cancer Hospital, Zhengzhou, \\ Henan, China \\ 3 Department of Oncology, Yueyang Hospital of Integrated Traditional Chinese and Western Medicine, Shanghai University of Traditional Chinese Medicine \\ (TCM), Shanghai, China \\ 4 Department of General Surgery, Renji Hospital, Shanghai Jiao Tong University School of Medicine, Shanghai, China \\ Corresponding Author: Aiguang Zhao \\ Email address: aiguangzhao@qq.com
}

BACKGROUND: Gastric cancer (GC) is a heterogeneous disease that encompasses various molecular subtypes. The molecular mutation characteristics of circulating tumor DNA (ctDNA) in advanced gastric cancer (AGC), especially the clinical utility of TP53 mutation and MET amplification in ctDNA need to be further explored.

OBJECTIVES: The aim of this study was mainly to assess the clinical utility of TP53 mutation and MET amplification in ctDNA as biomarkers for monitoring disease progression of AGC.

PATIENTS AND METHODS: We used multigene NGS-panel technology to study the characteristics of ctDNA gene mutations and screen the key mutant genes in AGC patients. Kaplan-Meier method was used to calculate the survival probability and log-rank test was used to compare the survival curves of TP53 mutation and MET amplification in ctDNA of AGC patients. The survival time was set from the blood test time to the follow-up time to observe the relationship between the monitoring index and tumor prognosis.

RESULTS: We performed mutation detection on ctDNA in 23 patients with AGC and identified the top 20 mutant genes. The five most frequently mutated genes were TP53 (55\%), EGFR (20\%), ERBB2 (20\%), MET (15\%) and APC (10\%). TP53 was the most common mutated gene $(55 \%)$ and MET had a higher frequency of mutations (15\%) in our study. Kaplan-Meier analysis showed that patients with TP53 mutant in ctDNA had shorter overall survival (OS) than these with TP53 wild (P\0.001). The Allele frequency (AF) of TP53 mutations in patient number 1 was higher in the second time $(0.94 \%)$ than in the first time (0.36\%); the AF of TP53 mutations in patient number 16 was from scratch $(0 \sim 0.26 \%)$. In addition, the AF of TP53 mutations in patients who survive was relatively low $(P=0.047)$. Simultaneously, Kaplan-Meier analysis showed that patients with MET amplification also had shorter OS than these with MET without amplification $(P \square 0.001)$.

CONCLUSION: TP53 and MET are the two common frequently mutant genes in ctDNA of AGC patients.TP53 mutation and MET amplification in ctDNA could predict disease progression of AGC patients. 
1 Title: TP53 mutation and MET amplification in circulating tumor DNA analysis predict disease progression in

2 patients with advanced gastric cancer

3 Author order: Jia $\mathrm{Li}^{1,2 \#}$, Zhaoyan $\mathrm{Li}^{3 \#}$, Yajie Ding ${ }^{1}$, Yan $\mathrm{Xu}^{1}$, Xiaohong $\mathrm{Zhu}^{1}$, Nida $\mathrm{Cao}^{1}$, Chen Huang ${ }^{4}$,

4 Mengmeng Qin ${ }^{1}$, Feng Liu ${ }^{1}$, Aiguang Zhao ${ }^{1 *}$.

5 Author affiliations:

$6{ }^{1}$ Department of Oncology, Longhua Hospital, Shanghai University of Traditional Chinese Medicine (TCM),

7 Shanghai 200032, China.

$8 \quad{ }^{2}$ Department of Integrated Chinese and Western Medicine, Affiliated Cancer Hospital of Zhengzhou University

9 and Henan Cancer Hospital, Zhengzhou, Henan 450008, China.

$10{ }^{3}$ Department of Oncology, Yueyang Hospital of Integrated Traditional Chinese and Western Medicine, Shanghai

11 University of Traditional Chinese Medicine (TCM), Shanghai 200437, China.

$12{ }^{4}$ Department of General Surgery, Renji Hospital, Shanghai Jiao Tong University School of Medicine, Shanghai

13 200032, Shanghai, China.

\#Authors contributed equally to this work. Jia Li and Zhaoyan Li are first authors and contributed equally

to this work.

*Corresponding authors:

Aiguang Zhao M.D. Ph.D.

Senior Doctor/Professor 


\section{ABSTRACT:}

BACKGROUND: Gastric cancer (GC) is a heterogeneous disease that encompasses various molecular subtypes. The molecular mutation characteristics of circulating tumor DNA (ctDNA) in advanced gastric cancer (AGC), especially the clinical utility of TP53 mutation and MET amplification in ctDNA need to be further explored.

OBJECTIVES: The aim of this study was mainly to assess the clinical utility of TP53 mutation and MET amplification in ctDNA as biomarkers for monitoring disease progression of AGC.

PATIENTS AND METHODS: We used multigene NGS-panel technology to study the characteristics of ctDNA gene mutations and screen the key mutant genes in AGC patients. Kaplan-Meier method was used to calculate the survival probability and log-rank test was used to compare the survival curves of TP53 mutation and MET amplification in ctDNA of AGC patients. The survival time was set from the blood test time to the follow-up time to observe the relationship between the monitoring index and tumor prognosis.

RESULTS: We performed mutation detection on ctDNA in 23 patients with AGC and identified the top 20 mutant genes. The five most frequently mutated genes were TP53 (55\%), EGFR (20\%), ERBB2 (20\%), MET mutations in patient number 1 was higher in the second time $(0.94 \%)$ than in the first time $(0.36 \%)$; the AF of TP53 mutations in patient number 16 was from scratch $(0 \sim 0.26 \%)$. In addition, the AF of TP53 mutations in 
patients with MET amplification also had shorter OS than these with MET without amplification $(P<0.001)$.

44 CONCLUSION: TP53 and MET are the two common frequently mutant genes in ctDNA of AGC patients.TP53

mutation and MET amplification in ctDNA could predict disease progression of AGC patients.

KEYWORDS: Advanced gastric cancer, circulating tumor DNA, TP53 mutation, MET amplification, disease

progression.

\section{Introduction}

Gastric cancer is one of the most common malignancies in the world. There are one million new cases of gastric

cancer and 783,000 deaths each year in the world (Bray et al, 2018). There are about 679,000 new cases of

gastric cancer and the number of deaths is about 498,000 each year in china (Chen et al, 2016). Despite advances

in surgery-based comprehensive treatment, more than $30 \%$ patients with gastric cancer are diagnosed at an

advanced stage and about $50 \%$ of patients after surgery will relapse and metastasize (Peng et al, 2020; Zhao et

al, 2019; Zong et al, 2016). Therefore, over $80 \%$ of gastric cancer patients are in the advanced stage with poor

clinical outcome in china. Due to gastric cancer is a heterogeneous disease that encompasses various molecular

subtypes, personalized-medicine approaches that commonly rely on a single tumor-biopsy sample to portray

tumor mutational landscapes cannot achieve satisfactory treatment effect (Hu et al, 2012; Wong et al, 2014;

Gerlinger et al, 2012). It is urgently needed to circumvent the issues of intratumoral heterogeneity and the

limitations and risks related to the biopsy procedure.

Non-invasive circulating tumor DNA (ctDNA) sequencing, a method of liquid biopsy, provides a potential tool

for real-time monitoring of tumor disease progression (Wang et al, 2019). The ctDNA includes small fragments

of double-stranded DNA (160-180 base pairs) and carries tumor related genetic alterations released by apoptotic 
64 (Hamakawa et al, 2015). Many studies have described the use of ctDNA in advanced tumor, including advanced

gastric cancer (AGC) patients (Marzese et al, 2013; Creemers et al, 2017; Frankell \& Smyth, 2019). T Hamakawa

et al. reported that ctDNA may serve as a useful biomarker to monitor gastric cancer progression and residual disease (Hamakawa et al, 2015). Wu et al. also found that ctDNA could be used as an effective tool to monitor the efficacy of chemotherapy and predict prognosis in advanced gastric cancer (Wu et al, 2020). Jian Yang et al. demonstrated that ctDNA could detect molecular residual disease and monitor for disease recurrence in definitively-treated locoregional gastric cancer (Yang et al, 2020). With the development of multigene NGSpanel technology, targeted capture sequencing can cost-effectively detect gene regions to provide cancerassociated mutations in ctDNA, thereby enhancing the clinical implementation of mutation analysis (Meyerson et al, 2010; Metzker, 2010). Recent studies using NGS have shown that a wide range of potential cancer-driven genes have caused mutations in gastric cancer (Cai et al, 2019; Chen et al, 2015; Xu et al, 2014) .

TP53 is the most common mutated gene in gastric cancer, accounting for about 50\% (Bellini et al, 2012 ). TP53 has an important role in regulating cell proliferation processes and maintaining genomic integrity and stability (Ozaki \& Nakagawara, 2011; Lane \& Levine, 2010). MET is a proto-oncogene located on chromosome 7q2131 and encodes a hepatocyte growth factor (HGF) receptor and acts as a carcinogenic driver in tumors, resulting in extensive downstream effects on tumor growth, invasiveness, angiogenesis, epithelial-mesenchymal transition and metastasis (An et al, 2014; Gherardi et al, 2012; Kim et al, 2018).The relationship between TP53 and MET gene abnormalities (such as mutations and amplifications) and clinical pathology in gastric cancer tissues has been studied (Baniak et al, 2016; Fenoglio-Preiser et al, 2003; Peng et al, 2014). In addition, studies have reported that characteristics of genetic mutations in ctDNA and tumor tissue are inconsistent (Creemers et al, 2017; Frankell \& Smyth, 2019;Maron et al, 2019). Therefore, the molecular mutation characteristics of ctDNA in AGC 
and the clinical utility of TP53 mutation and MET amplification in ctDNA need to be further explored.

In this study, we used multigene NGS-panel technology to study the characteristics of ctDNA gene mutations in AGC patients. Then, we analyzed the clinical utility of TP53 mutation and MET amplification in ctDNA of AGC patients.

\section{Materials and methods}

\section{Patients and samples}

The 23 patients included in this study were all patients with stage IV advanced gastric cancer. Patients were all drawn from Longhua Hospital, Shanghai University of Traditional Chinese Medicine. This study was approved by the ethics committee of shanghai university of traditional chinese medicine (2017LCSY021) and was consistent with the Helsinki Declaration. All patients received written informed consent for this study. The peripheral blood plasma samples of 23 patients were collected, of which 5 patients collected two blood samples.

Therefore, a total of 28 blood samples were used to detect somatic mutations. Clinical characteristics of 23 AGC patients was also collected (Supplemental Material 1). The somatic mutations, somatic copy number alteration and clinical data of 224 AGC tissue samples were downloaded from the TCGA portal (https://tcgadata.nci.nih.gov/tcga/).

\section{DNA extraction and target capture sequencing}

According to the manufacturer's instructions, plasma DNA were extracted from blood samples by QIAamp DNA Blood Mini Kit (Qiagen, Hilden, Germany). Later, the concentration of plasma DNA was measured by Qubit Fluorometer and Qubit dsDNA HS Analysis Kit (Invitrogen, Carlsbad, CA, USA). Then, the fragment status was evaluated by Agilent 2200 TapeStation system and the DNA Integrity Number (DIN) was generated by ScreenTape assay (Agilent Technologies, Santa Clara, CA, USA). Finally, DNA was sheared prior to using an 
ultrasonoscope with a peak of $250 \mathrm{bp}$, followed by end repair.

Indexed Illumina NGS libraries were prepared from circulating DNA libraries were prepared using the KAPA

Library Preparation Kit (Kapa Biosystems, Wilmington, MA, USA). The panel for detection of ctDNA in peripheral blood plasma samples from patients, were designed to cover 471 hot-spot mutations of 197 genes (including $17 \mathrm{NCCN}$ guidelines-recommended tumor-associated genes and 180 common tumor burdenassociated genes).

Next, Target enrichment was performed using the customized SeqCap EZ library (Roche NimbleGen, Madison, Wis.) according to the manufacturer's protocol. DNA sequencing was performed with $2 \times 150 \mathrm{bp}$ paired-end reads on the HiSeq 3000 sequencing system (Illumina, Inc). The genes of panel are listed in supplemental Material 2.

\section{Identification of somatic mutation in ctDNA}

Terminal adaptor sequences and low-quality reads were removed from the raw data. The clean reads were aligned

to the human genome build GRCh37 using BWA software version 0.7.12 - r1039. Somatic single nucleotide variants (SNVs) and small insertions and deletions (Indels) were generated using MuTect version 1.1.4 and GATK version 3.4-46-gbc02625, respectively. CONTRA v2.0.8 was used to detect copy number variants (CNVs). The candidate variants were all manually verified in the Integrative Genomics Viewer (IGV). Candidate somatic mutations were SNVs and Indels where the variant allele fraction (VAF) was $\geq 2 \%$ and there were $\geq 5$ high-quality reads (Phred score $\geq 30$, mapping quality $\geq 13$, and without paired-end reads bias) containing the target base. Non-synonymous mutations annotated by ANNOVAR were used in clonal structure reconstruction.

\section{Research process analysis}

To screen for common mutations in patients with advanced gastric cancer, we first performed NGS sequencing analysis on plasma samples from 23 patients with advanced gastric cancer and obtained gene sequencing data. 
127 Then, we compared the obtained sequencing data with the genetic sequencing data of patients with advanced

128 gastric cancer in TCGA. Finally, we analyzed the TP53 gene mutation and MET gene amplification, and obtained

129 the significance of TP53 and MET in the gene mutation data of advanced gastric cancer. The flow chart of this

130 study was shown in Figure 1.

131 Statistical analysis

132 The mutation landscape across a cohort, including SNVs, InDels and mutational burden, were created by

133 Genomic Visualizations in R (GenVisR). Kaplan-Meier survival plots were generated for TP53 mutation and

134 MET amplification at baseline using log-rank tests. All statistical analyses were performed with SPSS (v.22.0,

IL, Chicago, USA) or GraphPad Prism (v. 6.0, GraphPad Software, Chicago, USA) software. All statistical tests

were bilateral and $P<0.05$ was considered statistically significant.

Result

138 Characteristics of patients and sequencing data

139 All 23 AGC patients included in this study, and their clinical characteristics were presented in Table 1. Fifteen

140 of them were older than 60 years and 20 were male. All the pathological types were adenocarcinoma, with 7

141 cases of liver metastasis and 4 cases of peritoneal metastasis. According to lauren classification (Tang et al,

142 2020), 5 cases were classified as diffuse-type and 18 other types. Of these patients, 12 had undergone radical

surgery, 4 had palliative resection, and 7 had no surgery. The cohort included 11 patients receiving chemotherapy

and 12 patients receiving supportive care only. When performing gene sequencing, 20 patients had tumor burden.

145 NGS sequencing results of 23 AGC patients were included in Supplemental Material 3.

146 Landscape of somatic mutations

147 We performed mutations of ctDNA in 23 plasma samples from AGC patients and identified the top 20 mutant 
148

149

150

151

152

153

154

155

156

157

genes (Figure 2A). The five most frequently mutated genes were TP53 (55\%), EGFR (20\%), ERBB2 (20\%), MET (15\%) and APC (10\%). Figure 2B shows the top 20 mutant genes of tumor tissue from 224 AGC patients in the TCGA database.TP53 was the most common mutated gene in TCGA (44\%) and our study (55\%). MET amplification had a higher frequency of mutations (15\%) in this study, while the frequency of mutations was very low in the TCGA database.

\section{Relationship between TP53 mutation and prognosis of patients with advanced gastric cancer}

In the TCGA database, based on the survival analysis of the TP53 status (TP53 mutant and wild type), the overall survival (OS) clinically was not statistically significant. As shown in Figure 3A and 3B, Kaplan-Meier survival analysis according to the TCGA database showed that TP53 status in early stage gastric cancer (stage I, II) and advanced gastric cancer (stage III, IV) was not associated with OS ( $P=0.57$ and $P=0.14)$.

In the analysis of ctDNA for our 23 plasma samples, the prognostic value of TP53 mutations was demonstrated.

Kaplan-Meier analysis showed that patients with TP53 mutant had shorter survival time than these with TP53 wild (Figure 3C,P<0.001). Since the period of follow-up was set from the blood test time to the end of followup (2018.9.30), its significance indicated the relationship between the monitoring index (TP53) and the tumor prognosis.

To illustrate the role of TP53 mutation in predicting disease progression, we observed 5 AGC patients with rapid disease progression, and performed ctDNA sequencing on their blood samples again. The time of blood sample collection was the time of imaging examination or clinically judged as disease progression. TP53 mutations were detected in only 3 patients, namely patients 1,16 , and 17 . No TP53 mutation was detected in the other two patients, patients 9 and 11 . Then, we compared the results of the second ctDNA sequencing of patients 1,16 , and 17 with the first sequencing results, and mainly analyzed the changes in the Allele Frequency (AF) of TP53. 
169 We found that the AF of TP53 mutations in patient 1 was higher in the second time $(0.94 \%)$ than in the first time

$170(0.36 \%)$; the AF of TP53 mutations in patient 16 was from scratch $(0 \sim 0.26 \%)$. Moreover, the AF of TP53 in

171 patient 17 ranged from 0 to $0.17 \%$, which is similar to that of patient 16 (Figure $3 \mathrm{D}$ ). It indicated that the AF of

172 TP53 mutations was positively correlated with tumor progression. Although TP53 mutations had also occurred

173 in patients who survive, it was clearly found that AF was relatively low (Figure 3E, $P=0.047$ ).

\section{Relationship between MET amplification and prognosis of patients with advanced gastric cancer}

In order to analyze the effect of MET amplification in tumor tissue on the prognosis of patients with AGC, we downloaded the data with MET amplification information in the TCGA database and found that MET amplification had a relationship with the survival of patients with gastric cancer. Kaplan-Meier survival analysis showed that patients with MET amplification had shorter overall survival than these with MET without amplification (Figure 4A, $P<0.001$ ).

We divided the stomach adenocarcinoma (TCGA) database into early stage gastric cancer (stage I, II) and advanced gastric cancer (stage III, IV). The MET amplification was $1.1 \%(2 / 179)$ in the early stage gastric cancer and $4.6 \%$ in the advanced gastric cancer $(10 / 217)(P=0.0732)$. Therefore, it can be seen that MET amplification was more common in advanced gastric cancer. Kaplan-Meier survival curves showed that disease/progressionfree survival (DFS/PFS) of patients with MET amplification in tumor tissue was shorter than the patients with MET without amplification (Figure 4B, $p=0.0078$ ). In the analysis of ctDNA for our 23 plasma samples, KaplanMeier analysis showed that patients with MET amplification also had shorter overall survival than these with MET without amplification (Figure 4C, $P<0.001$ ).

\section{Discussion}

Conventional tumor tissue biopsy techniques are not only invasive and harbor risk of complications, but not also 
190

191

192

193

194

suitable for repeated operations. In addition, due to the characteristics of tumor heterogeneity, we cannot obtain accurate tumor molecular profile (Parikh et al, 2019). Liquid biopsy techniques, such as ctDNA, can not only avoid these limitations, but have also been integrated into daily clinical practice (Ilie \& Hofman, 2016).

Multigene NGS-panel technology for assessing ctDNA can be used to study a variety of genes, while only a small amount of ctDNA is obtained from even smaller samples, eventually including circulating tumour cells and free serum DNA (Meyerson et al, 2010; Pan et al, 2018). Comparing with traditional technology (dPCR), NGS sequencing technology has high sensitivity and accuracy for detecting rare ctDNA mutations, thus detecting very rare mutations with $\mathrm{AF}<0.001 \%$ and representing the original DNA population (Kukita et al, 2015). Therefore, it is now possible to detect tumor-related mutations on ctDNA and monitor the evolution of cancer progression in patients (Bettegowda et al, 2014). Our present report mainly analyzed the evaluation of ctDNA mutations using multigene NGS-panel technology and exploring the clinical significance of TP53 mutation and MET amplification in ctDNA of AGC. We performed mutation detection on ctDNA in 23 patients with AGC and identified the top 20 mutant genes. The five most frequently mutated genes were TP53 (55\%), EGFR (20\%), ERBB2 (20\%), MET (15\%) and APC (10\%). TP53 was the most common mutated gene in TCGA (44\%) and our study (55\%). MET amplification had a higher frequency of mutations (15\%) in this study, while the frequency of mutations was very low in the TCGA database.

In fact, survival analysis of early gastric cancer or advanced gastric cancer in TCGA database show no significant clinical outcomes in overall survival (OS) based on TP53 status in tumor tissue, which is consistent with previous reports (Bellini et al, 2012). We demonstrated a good correlation between the TP53 status of ctDNA and the disease status of AGC. Kaplan-Meier analysis showed that patients with TP53 mutant had shorter overall survival than these with TP53 wild $(\mathrm{P}<0.001)$. Since the period of follow-up was set from the blood test time 
211 to the end of follow-up, its significance indicated the relationship between the monitoring index (TP53) and the

212 tumor prognosis. We also observed that the AF of TP53 mutation increased after tumor progression, indicating

213 its potential application for monitoring progressive disease. Although TP53 mutation also occurred in surviving

214 patients, it was clearly found that AF was relatively low. Therefore, the results of our study indicated that TP53

215 mutation might have potential application in predicting disease progression and be related to the AF of TP53

216 mutations.

217 Some studies have also investigated the potential of TP53 mutations in ctDNA in gastric cancer. One report

demonstrated that ctDNA could be used for monitoring gastric cancer disease progression. Cancer-associated

somatic mutations (such as TP53 mutations) are specific to malignancies, and DNA with these mutations

indicates the presence of malignancies (Hamakawa et al, 2015). The other study examined the role of ctDNA to

diagnose or monitor disease status in esophageal and gastric cancer. The allele frequency (AF) of the TP53

mutation was low at diagnosis and rapidly increased during disease progression. In contrast, the allele frequency

(AF) of TP53 in cfDNA showed no significant changes (Kukita et al, 2015). Focusing on the mutation analysis

in GC patients, ctDNA was demonstrated to be a useful predictor for treatment response. The AF of ctDNA

decreased after surgical resection and simultaneously increased with recurrent or progressive disease during

palliative treatment. A similar trend was seen in some cases, the AF of TP53 mutation remained low until

recurrence, at which point the AF increased to 3.6\% (Hamakawa et al, 2015). Furthermore, in two out of four

patients disease recurrence was detected first by ctDNA, with an increasing AF of TP53 earlier before

radiological confirmation (Ueda et al, 2016). Therefore, these studies demonstrated that TP53 may be related to 
232

mutations in ctDNA or cfDNA have also been reported. Chiaki Nakauchi et al. demonstrated that the patients with TP53 mutation in cfDNA showed a tendency toward a worse prognosis than those without it (Nakauchi et al, 2016). Parkinson et al retrospectively analyzed TP53 mutations in cfDNA as biomarker of treatment response for patients with relapse high-grade serous ovarian carcinoma and demonstrated that a decrease of $\leq 60 \%$ in TP53 mutant allele fraction after one cycle of chemotherapy was associated with shorter time to progression (Parkinson et al, 2016). Kim Y M et al. also found that TP53 mutations in ctDNA was useful as a non-invasive biomarker of treatment response monitoring in patients with high-grade serous ovarian carcinoma (Kim et al, 2019). Hakan Savlia et al. demonstrated that TP53, EGFR and PIK3CA gene variations observed as prominent biomarkers in breast and lung cancer by plasma cell-free DNA genomic testing and were recommended as useful biomarkers for predictive studies to follow up tumor growth (Savli et al, 2019). Taryn D. Treger et al. demonstrated for the first time that ddPCR was an effective method for detection of mutant TP53 in ctDNA from children with anaplastic wilm' tumor, even when there was intratumoral somatic heterogeneity (Treger et al, 2018). In conclusion, TP53 mutation in ctDNA is the most common in various tumors, the significant clinical of TP53 mutations impact on future risk stratification and surveillance should be further explored in a larger cohort of patients.

We also further explored the prognostic value of MET amplification in the TCGA database and the ctDNA of AGC patients, Kaplan-Meier survival curves showed that OS and DFS/PFS of GC patients with MET amplification was shorter than the patients with MET without amplification $(P<0.001$ and $P=0.0078)$. The MET amplification of advanced gastric cancer was $4.6 \%$ (10/217) higher than that of early gastric cancer $1.1 \%(2 / 179)$, although not statistically significant $(P=0.0732)$. A systematic review and meta-analysis showed that MET overexpression and gene amplification in tumor tissue were indicators of poor prognosis in patients with gastric 
253

254

255

256

257

258

259

260

261

262

263

264

265

266

267

268

269

270

271

272

273

cancer (Peng et al, 2014). Other data show that $8.3 \%$ of patients with metastatic gastric cancer exhibit MET

amplification, and at least in the Chinese population, MET amplification is more frequent in patients with recurrent/metastatic gastric cancer (Lane \& Levine, 2010). These findings suggest that MET amplification is not common and may play a key role in advanced gastric cancer.

The detection of MET amplification in ctDNA is still scarce, and only one study has been reported

MET alterations detected in ctDNA so far. The study demonstrated that assessment of MET genomic aberrations by liquid biopsy is feasible and found that MET ctDNA anomalies were associated with bone metastases, multiple genomic alterations, and a worse prognosis, including shorter overall survival and a shorter time to recurrence/metastases (Ikeda et al, 2018). In our study, the survival rate of patients with advanced gastric cancer with MET amplification was significantly reduced. Therefore, MET amplification in ctDNA may predict disease progression in patients with advanced gastric cancer in the same way as MET amplification in tumor tissues.

In conclusion, TP53 and MET are the two common frequently mutant genes in ctDNA of AGC patients. TP53 may be related to the disease progression of AGC patients, in a manner that may be related to the AF of TP53 mutations. MET amplification in ctDNA may predict disease progression in patients with AGC in the same way as MET amplification in tumor tissues. This study still has some limitations. Firstly, the sample size for ctDNA testing in this study was limited, a multivariate analysis was not conducted. Secondly, we did not analyze the treatment status of the included cases (such as chemotherapy), although they also had a certain impact on the results of ctDNA. Therefore, the clinical utility of TP53 mutation and MET amplification in ctDNA impact on future risk stratification and surveillance should be further explored in a larger cohort of patients.

\section{Acknowledgements}

Peer] reviewing PDF | (2020:07:50937:2:0:NEW 3 Feb 2021) 
274 Not applicable.

\section{Conflict of Interest Statement}

276 All authors declare that there is no conflict of interest.

277

278

279

280

281

282

283

284

285

286

287

288

289

290

291

292

293

294

295

296

\section{Role of the funding source}

The research was supported by the Ministry of Science and Technology National Key Research and Development Program (2017YFC1700605), the National Health and Family Planning Commission's "Major New Drug Creation" Science and Technology Major Project (2017ZX09304-001) and the National Traditional Chinese Medicine Administration National TCM Clinical Research Base Business Construction Research Project (JDZX2015068). Key R\&D and promotion projects in Henan Province (212102310342).

\section{Ethical approval and informed consent}

This study was approved by the Ethics Committee of Shanghai University of Traditional Chinese Medicine (Shanghai, China) and was consistent with the Helsinki Declaration. The reference number for the ethics approval statement is 2017LCSY021. All patients provided written informed consent to participate in the study.

\section{Reference}

An X,Wang F,Shao Q,Wang FH,Wang ZQ,Wang ZQ,Chen C,Li C,Luo HY,Zhang DS,Xu RH and Li YH. 2014. MET amplification is not rare and predicts unfavorable clinical outcomes in patients with recurrent/metastatic gastric cancer after chemotherapy. CANCER 120:675-682. DOI 10.1002/cncr.28454.

Bray F, Ferlay J, Soerjomataram I, Siegel RL, Torre LA, and Jemal A. 2018. Global cancer statistics 2018: GLOBOCAN estimates of incidence and mortality worldwide for 36 cancers in 185 countries. CA Cancer J Clin 68:394-424. DOI 10.3322/caac.21492.

Bellini MF,Cadamuro AC,Succi M,Proenca MA,and Silva AE. 2012. Alterations of the TP53 gene in gastric and esophageal carcinogenesis. J Biomed Biotechnol 2012:891961. DOI 10.1155/2012/891961.

Baniak N,Senger JL,Ahmed S,Kanthan SC,and Kanthan R. 2016. Gastric biomarkers: a global review. World 
297

298

299

300

301

302

303

304

305

306

307

308

309

310

Journal of Surgical Oncology 14:212. DOI 10.1186/s12957-016-0969-3.

Bettegowda C,Sausen M,Leary RJ,Kinde I,Wang Y,Agrawal N,Bartlett BR,Wang H,Luber B,Alani RM,Antonarakis ES,Azad NS,Bardelli A,Brem H,Cameron JL,Lee CC,Fecher LA,Gallia GL,Gibbs P,Le D, Giuntoli RL,Goggins M,Hogarty MD,Holdhoff M,Hong SM,Jiao Y,Juhl HH,Kim JJ,Siravegna G,Laheru DA,Lauricella C,Lim M,Lipson EJ,Marie SK,Netto GJ,Oliner KS,Olivi A,Olsson L,Riggins GJ,Sartore-Bianchi A,Schmidt K,Shih L,Oba-Shinjo SM,Siena S, Theodorescu D, Tie J,Harkins TT, Veronese S, Wang TL, Weingart JD, Wolfgang CL, Wood LD,Xing D,Hruban RH,Wu J,Allen PJ,Schmidt CM,Choti MA,Velculescu VE,Kinzler KW,Vogelstein B,Papadopoulos N and Diaz LJ. 2014. Detection of circulating tumor DNA in early- and latestage human malignancies. Science Translational Medicine 6:224r. DOI 10.1126/scitranslmed.3007094.

Bellini MF,Cadamuro AC,Succi M,Proenca MA and Silva AE. 2012. Alterations of the TP53 gene in gastric and esophageal carcinogenesis. J Biomed Biotechnol 2012:891961. DOI 10.1155/2012/891961.

Chen W, Zheng R, Baade PD, Zhang S, Zeng H, Bray F, Jemal A, Yu XQ, and He J. 2016. Cancer statistics in China, 2015. CA Cancer J Clin 66:115-132. DOI 10.3322/caac.21338.

Creemers A,Krausz S,Strijker M,vanderWel MJ,Soer EC,Reinten RJ,Besselink MG,Wilmink JW,vande Vijver MJ,van Noesel C,Verheij J,Meijer SL,Dijk F,Bijlsma MF,van Oijen M and van Laarhoven H. 2017. Clinical value of ctDNA in upper-GI cancers: A systematic review and meta-analysis. Biochim Biophys Acta Rev Cancer 1868:394-403. DOI 10.1016/j.bbcan.2017.08.002.

Cai H,Jing C,Chang X,Ding D,Han T, Yang J,Lu Z,Hu X,Liu Z, Wang J,Shang L,Wu S,Meng P,Lin L,Zhao J,Nie M and Yin K. 2019. Mutational landscape of gastric cancer and clinical application of genomic profiling based on target next-generation sequencing. Journal of Translational Medicine 17:189. DOI 10.1186/s12967-0191941-0.

Chen K, Yang D,Li X,Sun B,Song F,Cao W,Brat DJ,Gao Z,Li H,Liang H,Zhao Y,Zheng H,Li M,Buckner J,Patterson SD,Ye X,Reinhard C,Bhathena A,Joshi D,Mischel PS,Croce CM,Wang YM,Raghavakaimal S,Li H,Lu X,Pan Y,Chang H,Ba S,Luo L,Cavenee WK,Zhang W and Hao, X. 2015. Mutational landscape of gastric adenocarcinoma in Chinese: implications for prognosis and therapy. Proc Natl Acad Sci U S A 112:1107-1112. DOI 10.1073/pnas.1422640112.

Diaz LJ, and Bardelli A. 2014. Liquid biopsies: genotyping circulating tumor DNA. JOURNAL OF CLINICAL 
ONCOLOGY 32:579-586. DOI 10.1200/JCO.2012.45.2011.

325 Fenoglio-Preiser CM,Wang J,Stemmermann GN and Noffsinger A. 2003. TP53 and gastric carcinoma: a review.

326

327

328

329

330

331

332

333

334

335

336

337

338

339

340

341

342

343

344

345

346

347

348

349

350

HUMAN MUTATION 21:258-270. DOI 10.1002/humu.10180.

Frankell AM,and Smyth EC. 2019. ctDNA in Gastric and Gastroesophageal Cancer: Prognostic, Predictive, or Preliminary? CLINICAL CANCER RESEARCH 25:6893-6895. DOI 10.1158/1078-0432.CCR-19-2774.

Gerlinger M, Rowan AJ, Horswell S, Math M, Larkin J, Endesfelder D, Gronroos E, Martinez P, Matthews N,Stewart A,Tarpey P,Varela I,Phillimore B,Begum S,McDonald NQ,Butler A,Jones D,Raine K,Latimer C,Santos CR,Nohadani M,Eklund AC,Spencer-Dene B,Clark G,Pickering L,Stamp G,Gore M,Szallasi Z,Downward J,Futreal PA,and Swanton C. 2012. Intratumor heterogeneity and branched evolution revealed by multiregion sequencing. N Engl J Med 366:883-892. DOI 10.1056/NEJMoa1113205.

Gherardi E,Birchmeier W,Birchmeier C and Vande WG. 2012. Targeting MET in cancer: rationale and progress. NATURE REVIEWS CANCER 12:89-103. DOI 10.1038/nrc3205.

Hu B, El HN, Sittler S, Lammert N, Barnes R, and Meloni-Ehrig, A. 2012. Gastric cancer: Classification, histology and application of molecular pathology. J Gastrointest Oncol 3:251-261. DOI 10.3978/j.issn.20786891.2012 .021

Hamakawa T,Kukita Y,Kurokawa Y,Miyazaki Y,Takahashi T,Yamasaki M,Miyata H,Nakajima K,Taniguchi K,Takiguchi S,Mori M,Doki Y,and Kato K. 2015. Monitoring gastric cancer progression with circulating tumour DNA. Br J Cancer 112:352-356. DOI 10.1038/bjc.2014.609.

Ilie M and Hofman P. 2016. Pros: Can tissue biopsy be replaced by liquid biopsy? Transl Lung Cancer Res 5:420-423. DOI 10.21037/tlcr.2016.08.06.

Ikeda S,Schwaederle M,Mohindra M,Fontes JD, and Kurzrock R. 2018. MET alterations detected in bloodderived circulating tumor DNA correlate with bone metastases and poor prognosis. Journal of Hematology \& Oncology 11:76. DOI 10.1186/s13045-018-0610-8.

Kim HS,Chon HJ,Kim H,Shin SJ,Wacheck V,Gruver AM,Kim JS,Rha SY and Chung HC. 2018. MET in gastric cancer with liver metastasis: The relationship between MET amplification and Met overexpression in primary stomach tumors and liver metastasis. JOURNAL OF SURGICAL ONCOLOGY 117:1679-1686. DOI 10.1002/jso.25097.

Peer] reviewing PDF | (2020:07:50937:2:0:NEW 3 Feb 2021) 
351 Kukita Y,Matoba R,Uchida J,Hamakawa T,Doki Y,Imamura F and Kato K. 2015. High-fidelity target 352 sequencing of individual molecules identified using barcode sequences: de novo detection and absolute 353 quantitation of mutations in plasma cell-free DNA from cancer patients. DNA RESEARCH 22:269-277. DOI $354 \quad 10.1093 /$ dnares/dsv010.

355 Kukita Y,Matoba R,Uchida J,Hamakawa T,Doki Y,Imamura F and Kato K. 2015. High-fidelity target 356 sequencing of individual molecules identified using barcode sequences: de novo detection and absolute 357 quantitation of mutations in plasma cell-free DNA from cancer patients. DNA RESEARCH 22:269-277. DOI 10.1093/dnares/dsv010.

359 Kim, YM,Lee SW,Lee YJ,Lee HY,Lee JE and Choi EK. 2019. Prospective study of the efficacy and utility of 360 TP53 mutations in circulating tumor DNA as a non-invasive biomarker of treatment response monitoring in 361 patients with high-grade serous ovarian carcinoma. Journal of Gynecologic Oncology 30:e32. DOI $362 \quad 10.3802 /$ jgo.2019.30.e32.

363 Lane D and Levine A. 2010. p53 Research: the past thirty years and the next thirty years. Cold Spring Harb 364 Perspect Biol 2:a893. DOI 10.1101/cshperspect.a000893.

365 Marzese DM,Hirose H,and Hoon DS. 2013. Diagnostic and prognostic value of circulating tumor-related DNA 366 in cancer patients. EXPERT REVIEW OF MOLECULAR DIAGNOSTICS 13:827-844. DOI $367 \quad 10.1586 / 14737159.2013 .845088$.

368 Meyerson M,Gabriel S,and Getz G. 2010. Advances in understanding cancer genomes through second369 generation sequencing. NATURE REVIEWS GENETICS 11:685-696. DOI 10.1038/nrg2841.

370 Metzker ML. 2010. Sequencing technologies - the next generation. NATURE REVIEWS GENETICS 11:31371 46. DOI 10.1038/nrg2626.

372 Maron SB,Chase LM,Lomnicki S,Kochanny S,Moore KL,Joshi SS,Landron S,Johnson J,Kiedrowski LA,Nagy RJ,Lanman RB,Kim ST,Lee Jand Catenacci D. 2019. Circulating Tumor DNA Sequencing Analysis of 374 Gastroesophageal Adenocarcinoma. CLINICAL CANCER RESEARCH 25:7098-7112. DOI 10.1158/1078375 0432.CCR-19-1704.

376 Meyerson M, Gabriel S, and Getz G. 2010. Advances in understanding cancer genomes through second377 generation sequencing. NATURE REVIEWS GENETICS 11:685-696.DOI 10.1038/nrg2841. 
378 Nadauld LD and Ford JM. 2013. Molecular profiling of gastric cancer: toward personalized cancer medicine.

379 JOURNAL OF CLINICAL ONCOLOGY 31:838-839. DOI 10.1200/JCO.2012.47.1714.

380 Ozaki T and Nakagawara A. 2011. p53: the attractive tumor suppressor in the cancer research field. J Biomed 381 Biotechnol 2011:603925. DOI 10.1155/2011/603925.

382 Nakauchi C,Kagara N,Shimazu K,Shimomura A,Naoi Y,Shimoda M,Kim SJ and Noguchi S. 2016. Detection 383 of TP53/PIK3CA Mutations in Cell-Free Plasma DNA From Metastatic Breast Cancer Patients Using Next 384 Generation Sequencing. Clinical Breast Cancer 16:418-423. DOI 10.1016/j.clbc.2016.05.004.

385 Peng W, Zhang F, Wang Z, Li D, He Y, Ning Z, Sheng L, Wang J, Xia X, Yu C, Wang Z, Zhao Y, Liang H, Hu 386 B, Sun C, Wang D, Cheng Y, Pan M, Xia L, Guo X, Zhang Y, Hu Z, Li X, Lu L, Zhang J, Qian H, Xie H, and 387 Sun G. 2020. Large Scale, Multicenter, Prospective Study of Apatinib in Advanced Gastric Cancer: A Real388 World Study from China. Cancer Management and Research 12:6977-6985. DOI 10.2147/CMAR.S249153.

389 Peng Z,Zhu Y,Wang Q,Gao J,Li Y,Li Y,Ge S and Shen L. 2014. Prognostic significance of MET amplification 390 and expression in gastric cancer: a systematic review with meta-analysis. PLoS One 9:e84502. DOI 391 10.1371/journal.pone.0084502.

392 Parikh AR,Leshchiner I,Elagina L,Goyal L,Levovitz C,Siravegna G,Livitz D,Rhrissorrakrai K,Martin E.E,Van 393 Seventer EE,Hanna M,Slowik K,Utro F,Pinto CJ,Wong A,Danysh BP,de la Cruz FF,Fetter IJ,Nadres 394 B,Shahzade HA,Allen JN,Blaszkowsky LS,Clark JW,Giantonio B,Murphy JE,Nipp RD,Roeland E,Ryan 395 DP,Weekes CD,Kwak EL,Faris JE,Wo JY,Aguet F,Dey-Guha I,Hazar-Rethinam M,Dias-Santagata D,Ting DT,Zhu AX,Hong TS,Golub TR,Iafrate AJ,Adalsteinsson VA,Bardelli AParida L,Juric D,Getz G and Corcoran RB. 2019. Liquid versus tissue biopsy for detecting acquired resistance and tumor heterogeneity in gastrointestinal cancers. NATURE MEDICINE 25:1415-1421. DOI 10.1038/s41591-019-0561-9.

Pan X,Ji X,Zhang R,Zhou Z,Zhong Y,Peng W,Sun N,Xu X,Xia L,Li P,Lu J and Tu J. 2018. Landscape of somatic mutations in gastric cancer assessed using next-generation sequencing analysis. Oncology Letters 16:4863-4870. DOI 10.3892/ol.2018.9314.

Parkinson CA,Gale D,Piskorz AM,Biggs H,Hodgkin C,Addley H,Freeman S,Moyle P,Sala E,Sayal K,Hosking 
405

406

407

408

409

410

411

412

413

414

415

416

417

418

419

420

421

422

423

424

425

426

427

428

429

430

431

High-Grade Serous Ovarian Carcinoma: A Retrospective Study. PLOS MEDICINE 13:e1002198. DOI 10.1371/journal.pmed.1002198.

Savli H,Sertdemir N,Aydin D,Dursun B,Kurtas O,Reka S,Sunnetci-Akkoyunlu D,Eren-Keskin S,Uygun K,Ozden E,Isik U,Cabuk D,Demir G,Guzdolu E,Gokbayrak M,Aksu MG,Canturk NZ and Cine N. 2019. TP53, EGFR and PIK3CA gene variations observed as prominent biomarkers in breast and lung cancer by plasma cell-free DNA genomic testing. JOURNAL OF BIOTECHNOLOGY 300:87-93. DOI 10.1016/j.jbiotec.2019.05.005.

Tang, C.T, Zeng, L, Yang, J, Zeng, C, and Chen, Y. 2020. Analysis of the Incidence and Survival of Gastric Cancer Based on the Lauren Classification: A Large Population-Based Study Using SEER. Frontiers in Oncology 10:1212. DOI 10.3389/fonc.2020.01212

Treger TD,Chagtai T,Butcher R,Cresswell GD,Al-Saadi R,Brok J,Williams RD,Roberts C,Luscombe NM,Pritchard JK and Mifsud W. 2018. Somatic TP53 Mutations Are Detectable in Circulating Tumor DNA from Children with Anaplastic Wilms Tumors. Translational Oncology 11:1301-1306. DOI 10.1016/j.tranon.2018.08.006.

Ueda M,Iguchi T,Masuda T,Nakahara Y,Hirata H,Uchi R,Niida A,Momose K,Sakimura S,Chiba K,Eguchi H,Ito S,Sugimachi K,Yamasaki M,Suzuki Y,Miyano S,Doki Y,Mori M and Mimori K. 2016. Somatic mutations in plasma cell-free DNA are diagnostic markers for esophageal squamous cell carcinoma recurrence. Oncotarget 7:62280-62291. DOI 10.18632/oncotarget.11409.

Wong SS, Kim KM, Ting JC, Yu K, Fu J, Liu S, Cristescu R, Nebozhyn M, Gong L, Yue YG, Wang J, Ronghua C, Loboda A, Hardwick J, Liu X, Dai H, Jin JG, Ye XS, Kang SY, Do IG, Park JO, Sohn TS, Reinhard C, Lee J, Kim S, and Aggarwal A. 2014. Genomic landscape and genetic heterogeneity in gastric adenocarcinoma revealed by whole-genome sequencing. Nature Communications 5:5477. DOI 10.1038/ncomms6477.

Wang, Y, Zhao C,Chang L,Jia R,Liu R,Zhang Y,Gao X,Li J,Chen R,Xia X,Bulbul A,Husain H,Guan Y,Yi X and Xu J. 2019. Circulating tumor DNA analyses predict progressive disease and indicate trastuzumab-resistant mechanism in advanced gastric cancer. EBioMedicine 43:261-269. DOI 10.1016/j.ebiom.2019.04.003.

Wu R,Shi C,Chen Q,Wu F and Li Q. 2020. Detection of circulating tumor cell DNA for monitoring advanced gastric cancer. Int J Clin Exp Pathol 13:203-211.

Peer] reviewing PDF | (2020:07:50937:2:0:NEW 3 Feb 2021) 
432 Xu Z,Huo X,Ye H,Tang C,Nandakumar V,Lou F,Zhang D,Dong H,Sun H,Jiang S,Zhang G,Li Z,Dong Z,Guo

433 B,He Y,Yan C Wang L,Su Z,Li Y,Gu D,Zhang X,Wu X,Wei X,Hong L,Zhang Y,Yang J,Gong Y,Tang C,Jones

434 L,Huang XF,Chen SY and Chen J. 2014. Genetic mutation analysis of human gastric adenocarcinomas using 435 ion torrent sequencing platform. PLoS One 9:e100442. DOI 10.1371/journal.pone.0100442.

436 Yang J,Gong Y,Lam VK,Shi Y,Guan Y,Zhang Y,Ji L,Chen Y,Zhao Y,Qian F,Chen J,Li P,Zhang F, Wang 437 J,Zhang X,Yang L,Kopetz S,Futreal PA,Zhang J,Yi X,Xia Xand Yu P. 2020. Deep sequencing of circulating 438 tumor DNA detects molecular residual disease and predicts recurrence in gastric cancer. Cell Death \& Disease 439 11:346. DOI 10.1038/s41419-020-2531-z.

440 Zhao L, Li J, Bai C, Nie Y, and Lin G. 2019. Multi-Modality Treatment for Patients With Metastatic Gastric 441 Cancer: A Real-World Study in China. Frontiers in Oncology 9:1155. DOI 10.3389/fonc.2019.01155.

442 Zong L, Abe M, Seto Y, and Ji J. 2016. The challenge of screening for early gastric cancer in China. LANCET 443 388:2606. DOI 10.1016/S0140-6736(16)32226-7.

Figure legends

446 Figure 1. The flow chart of this study.

447 Figure 2. Landscape of somatic mutations in AGC. (A) The genes and frequencies of ctDNA mutations in 23 448 plasma samples. (B) The genes and frequencies of 224 AGC sample mutations in the TCGA database.

449 Figure 3. Relationship between TP53 and prognosis of patients with advanced gastric cancer. (A) Kaplan-Meier 450 survival analysis of OS based on TP53 status in TCGA database of early stage gastric cancer (stage I, II). (B)

455 Figure 4. Relationship between MET amplification and prognosis of patients with advanced gastric cancer. (A) 
456 Kaplan-Meier survival analysis of OS based on MET amplification in TCGA database of gastric cancer. (B)

457 Kaplan-Meier survival analysis of DFS/PFS based on MET amplification in TCGA database of gastric cancer.

458 (C) Kaplan-Meier analysis of survival time based on MET amplification according to the clinical data of AGC

459 patients.

460 Table legends

461 Table 1: The characteristics of 23 AGC patients detected by ctDNA.

462

463 


\section{Table $\mathbf{1}$ (on next page)}

Table 1: The characteristics of 23 AGC patients detected by ctDNA. 
1

2 Table 1

3

\begin{tabular}{ll}
\hline Variables & Number (\%) \\
\hline Age & \\
$<60$ & $8(34.8)$ \\
$\geq 60$ & $15(65.2)$ \\
Sex & \\
$\quad$ Female & $3(13.0)$ \\
$\quad$ Male & $20(87.0)$ \\
Lauren classification & \\
Diffuse & $5(21.7)$ \\
Others & $18(78.3)$ \\
Liver metastasis & \\
$\quad$ Yes & $7(30.4)$ \\
No & $16(69.6)$ \\
Peritoneal metastasis & \\
Yes & $4(17.4)$ \\
No & $19(82.6)$ \\
Surgical approach & \\
Radical surgery & $12(52.2)$ \\
Palliative resection & $4(17.4)$ \\
Others & $7(30.4)$ \\
chemotherapy & \\
Yes & $11(47.8)$ \\
No & $12(52.2)$ \\
Tumor burden & \\
Yes & $20(87.0)$ \\
No & $3(13.0)$ \\
\hline
\end{tabular}


Figure 1

Figure 1. The flow chart of this study .

\section{Screening for key mutant genes}

NGS sequencing data for plasma samples from 23 AGC patients

Sequencing data of 224 AGC tissue samples in TCGA database

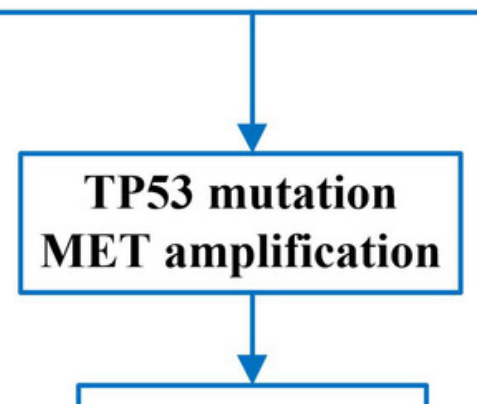

Data analysis 
Figure 2

Figure 2. Landscape of somatic mutations in AGC. (A) The genes and frequencies of ctDNA mutations in 23 plasma samples. (B) The genes and frequencies of 224 AGC sample mutations in the TCGA database.
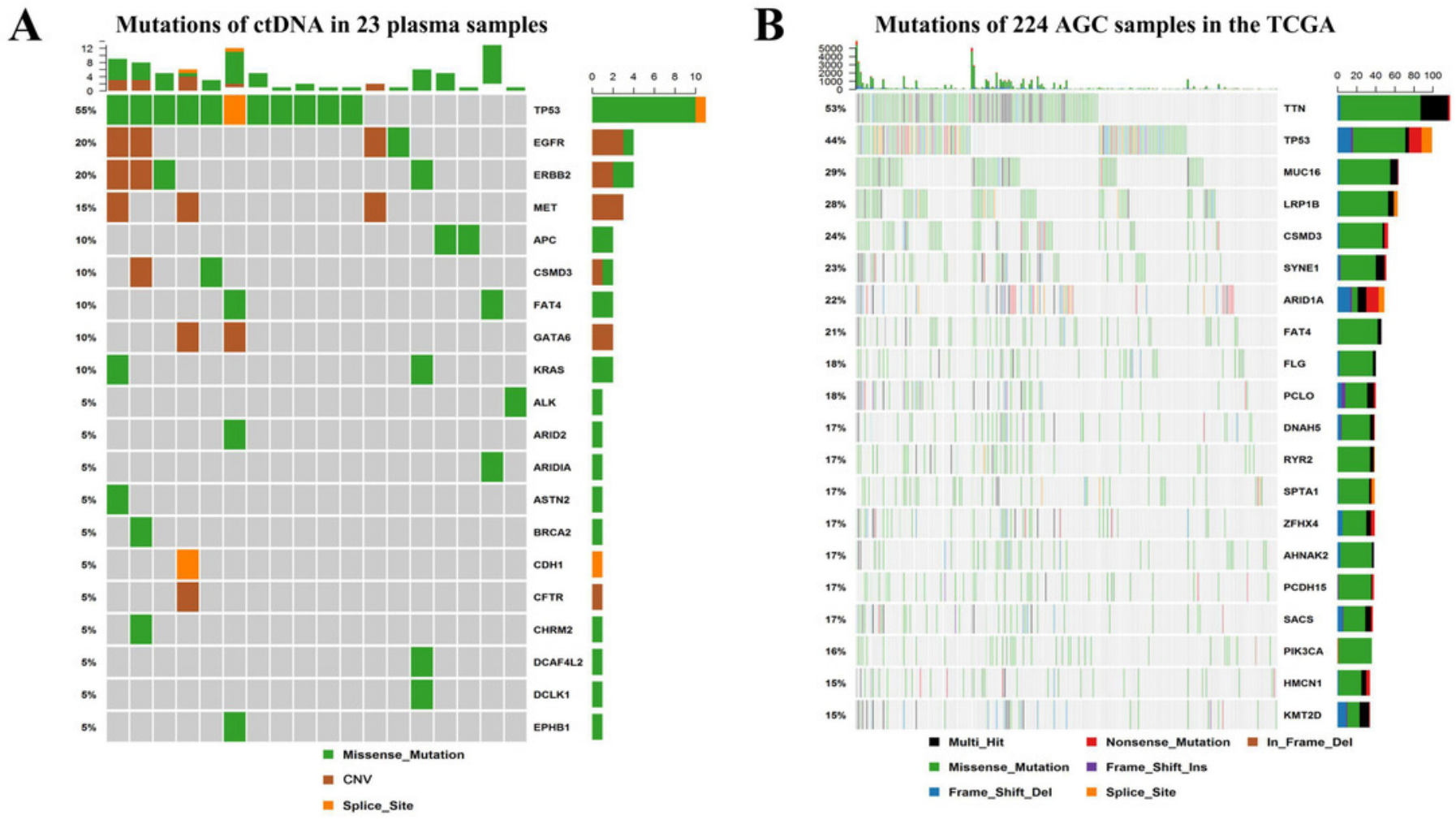
Figure 3

Figure 3. Relationship between TP53 and prognosis of patients with advanced gastric cancer. (A) Kaplan-Meier survival analysis of OS based on TP53 status in TCGA database of early stage gastric cancer (stage I, II). (B) Kaplan-Meier survival analysis of $O$

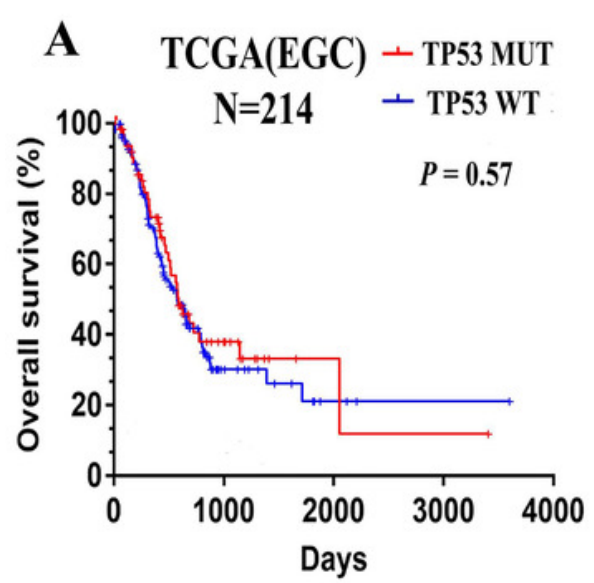

D

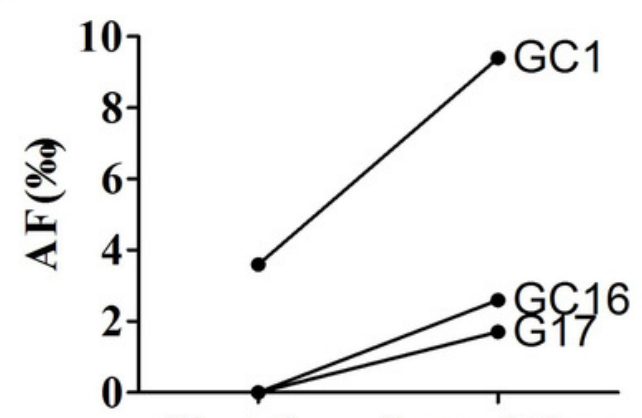

First time Second time ctDNA
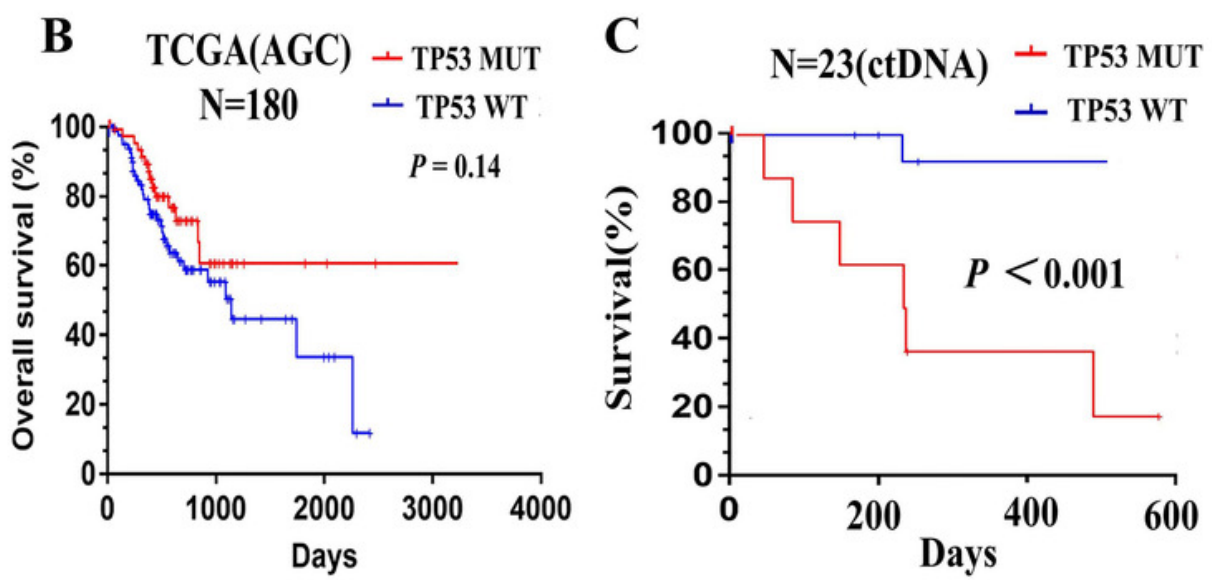

$P=0.047$ 
Figure 4

Figure 4. Relationship between MET amplification and prognosis of patients with advanced gastric cancer. (A) Kaplan-Meier survival analysis of OS based on MET amplification in TCGA database of gastric cancer. (B) Kaplan-Meier survival analysis of DFS/PFS

A

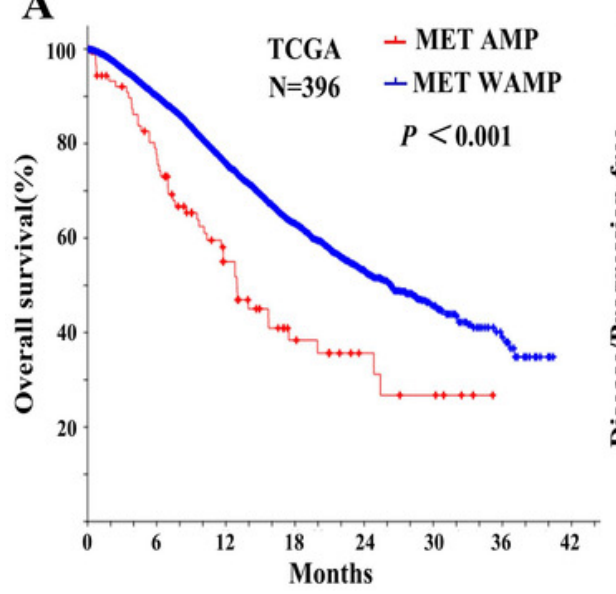

B

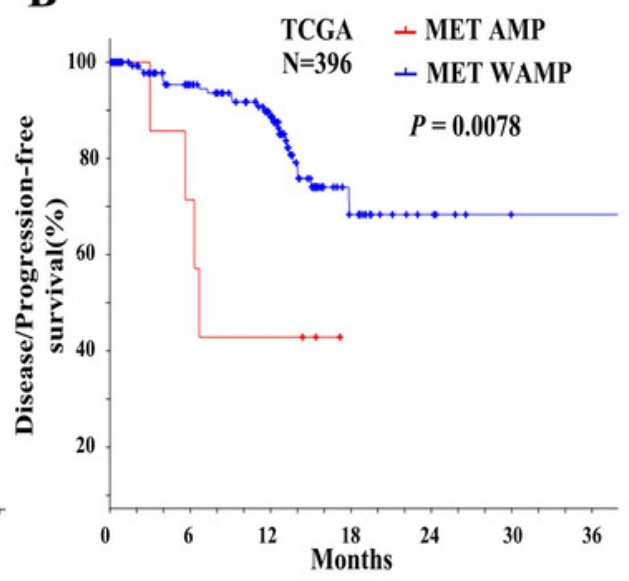

C

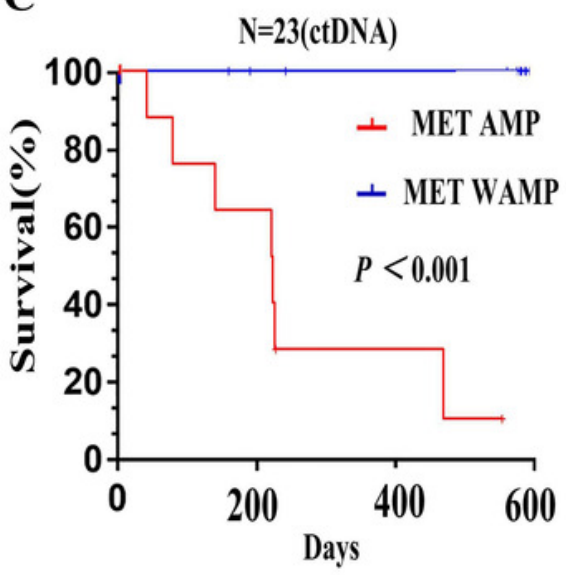

